\title{
Relations between the Energy of Graphs and Other Graph Parameters
}

\author{
Slobodan Filipovski*a \\ a FAMNIT, University of Primorska, Glagoljaška 8, 6000 Koper, Slovenia \\ slobodan.filipovski@famnit.upr.si
}

(Received September 8, 2021)

\begin{abstract}
In this paper we give various relations between the energy of graphs and other graph parameters as Randić index, clique number, number of vertices and edges, maximum and minimum degree etc. Moreover, new bounds for the energy of complementary graphs are derived. Our results are based on the concept of vertex energy developed by G. Arizmendi and O. Arizmendi in [Lin. Algebra Appl. doi:10.1016/j.laa.2020.09.025].
\end{abstract}

\section{Introduction}

Let $G=(V, E)$ be a simple undirected graph with vertex set $V(G)=\left\{v_{1}, v_{2}, \ldots, v_{n}\right\}$ and edge set $E(G),|E(G)|=m$. The adjacency matrix $A=A(G)$ of the graph $G$ is the $n \times n$ matrix $\left[a_{i j}\right]$ with $a_{i j}=1$ if $v_{i}$ is connected to $v_{j}$, and $a_{i j}=0$ otherwise. The eigenvalues $\lambda_{1}, \lambda_{2}, \ldots, \lambda_{n}$ of the graph $G$ are the eigenvalues of its adjacency matrix $A$. Since $A$ is a symmetric matrix with zero trace, these eigenvalues are real and their sum is equal to zero.

The energy of $G$, denoted by $E(G)$, was for the first time defined by I. Gutman in [8] as the sum of the absolute values of its eigenvalues. Hence,

$$
E(G)=\sum_{i=1}^{n}\left|\lambda_{i}\right| .
$$

\footnotetext{
${ }^{*}$ Corresponding author. This work is supported in part by the Slovenian Research Agency (research program P1-0285 and research project N1-0210).
} 
The concept of the energy of graphs arose in theoretical chemistry, since it can be used to approximate the total $\pi$-electron energy of a molecule. For details check $[9-12,18]$. The first result concerning the energy of a graph with its order and size is the following upper bound obtained in 1971 by McClelland [18]:

$$
E(G) \leq \sqrt{2 m n}
$$

Since then, numerous other bounds for $E(G)$ were published, see [5, 13, 14, 17]. In [15] Koolen and Moulton improved the bound (2) as follows: If $2 m>n$ and $G$ is a graph with $n$ vertices and $m$ edges, then

$$
E(G) \leq \frac{2 m}{n}+\sqrt{(n-1)\left(2 m-\left(\frac{2 m}{n}\right)^{2}\right)} .
$$

In [18], McClelland also obtained a lower bound involving the graph's order $n$, its size $m$, and the determinant of its adjacency matrix:

$$
E(G) \geq \sqrt{2 m+n(n-1)|\operatorname{det}(A)|^{\frac{2}{n}}}
$$

Almost thirty years later, a simpler lower bound involving only the number of edges $m$ was obtained in [6]:

$$
E(G) \geq 2 \sqrt{m}
$$

In order to understand locally the properties of the graph, in [2] Arizmendi and Romero introduced a vertex energy, a refinement of the graph energy.

Definition 1.1 The energy of the vertex $v_{i}$ with respect to $G$, which is denoted by $E_{G}\left(v_{i}\right)$, or simply $E\left(v_{i}\right)$, is given by

$$
E_{G}\left(v_{i}\right)=|A(G)|_{i i}, \text { for } i=1, \ldots, n
$$

where $|A|=\left(A A^{*}\right)^{1 / 2}$ and $A$ is the adjacency matrix of $G$.

Using the above definition the energy of a graph could be expressed as a sum of the vertex energies of $G$, that is,

$$
E(G)=E\left(v_{1}\right)+\ldots+E\left(v_{n}\right) .
$$

This paper is inspired by the new bounds for the energy of a vertex, derived in $[3,4]$. In [4] was proven that, if $v_{i}$ and $v_{j}$ are connected vertices of a simple (undirected) graph 
$G$, then $E\left(v_{i}\right) E\left(v_{j}\right) \geq 1$ and $E\left(v_{i}\right)+E\left(v_{j}\right) \geq 2$, while in [3] was proven that $E\left(v_{i}\right) \leq$ $\sqrt{\operatorname{deg}\left(v_{i}\right)}$. Combining these inequalities with the inequalities between means, we derive various bounds for the graph energy. Namely, in Section 2 we give new relationships between the energy of a given graph and its Randić index, clique number, maximum and minimum degree. In Section 3 we treat the energy of the complements of the graphs. We show that some of our bounds are better than the existing bounds.

\section{Bounds for the energy of graphs}

\subsection{New upper bounds}

Let $G$ be a graph on $n$ vertices and $\operatorname{let} \operatorname{deg}(v)$ be the degree of the vertex $v$. The Randic index of $G$ is defined as

$$
R(G)=\sum_{(u, v) \in E(G)} \frac{1}{\sqrt{\operatorname{deg}(u) \cdot \operatorname{deg}(v)}} .
$$

Based on the concept of vertex energy in [4] G. Arizmendi and O. Arizmendi proved

$$
E(G) \geq 2 R(G)
$$

Following the same methodology, in [21], Yan et al. give the following result:

Theorem 2.1 [21] Let $G$ be a graph with Randić index $R(G)$ and maximum degree $\Delta(G)$. Then $E(G) \leq 2 \sqrt{\Delta(G)} R(G)$ and the equality holds if and only if $G$ is the union of the path $P_{2}$.

In the first result of this paper we apply the same technique as in the proof of Theorem 2.1 , restricted on the class of triangle-free graphs. Recall, an undirected graph in which no three vertices form a triangle of edges is called triangle-free graph. It is well-known that, if $G$ is a triangle-free graph on $n$ vertices and if $e=(u, v) \in E(G)$, then $\operatorname{deg}(u)+\operatorname{deg}(v) \leq n$. This inequality is used in the following theorem.

Theorem 2.2 Let $G$ be a triangle-free graph on $n$ vertices. Then

$$
E(G) \leq \sqrt{2 n} R(G)
$$


Proof. We have

$$
\begin{aligned}
& E(G)=\sum_{v \in V(G)} E(v)=\sum_{e=(u, v) \in E(G)}\left(\frac{E(u)}{\operatorname{deg}(u)}+\frac{E(v)}{\operatorname{deg}(v)}\right) \\
& \leq \sum_{e \in E(G)}\left(\frac{\sqrt{\operatorname{deg}(u)}}{\operatorname{deg}(u)}+\frac{\sqrt{\operatorname{deg}(v)}}{\operatorname{deg}(v)}\right)=\sum_{e \in E(G)}\left(\frac{1}{\sqrt{\operatorname{deg}(u)}}+\frac{1}{\sqrt{\operatorname{deg}(v)}}\right) \\
& \leq \sum_{e \in E(G)} \sqrt{2\left(\frac{1}{\operatorname{deg}(u)}+\frac{1}{\operatorname{deg}(v)}\right)} \leq \sqrt{2 n} R(G) .
\end{aligned}
$$

Remark 2.3 If $\Delta(G) \geq \frac{n}{2}$, then $E(G) \leq \sqrt{2 n} R(G) \leq 2 \sqrt{\Delta(G)} R(G)$, that is, the bound in Theorem 2.2 is better than the bound in Theorem 2.1. Note that the molecular graphs as one of the most studied graphs in chemical graph theory do not satisfy this condition since $\Delta \leq 4$

A clique in a graph $G$ is a complete subgraph of $G$. A clique is called maximum if it has maximum cardinality. The clique number of a graph $G$, denoted $\omega(G)$, is the order of a maximum clique of $G$. Caro [7] and Wei [20], showed that the degree sequence $d_{1} \geq d_{2} \geq \ldots \geq d_{n}$ of a graph $G$ of order $n$ gives rise to a lower bound on the clique number of $G$ :

$$
\omega(G) \geq \frac{1}{n-d_{1}}+\frac{1}{n-d_{2}}+\ldots+\frac{1}{n-d_{n}} .
$$

In the next result we give a relation between the energy and the clique number of a given graph.

Theorem 2.4 Let $G$ be a graph with $n$ vertices and clique number $\omega(G)$. Then

$$
E(G) \leq \frac{n}{2} \sqrt{n \cdot \omega(G)}
$$

Proof. Let $d_{1} \geq \ldots \geq d_{n}$ be the degree-sequence of $G$. From AM-GM, for each $i=1, \ldots, n$ we have

$$
\sqrt{d_{i}} \sqrt{n-d_{i}} \leq \frac{d_{i}+n-d_{i}}{2}=\frac{n}{2}
$$

Thus

$$
E(G) \leq \sqrt{d_{1}}+\ldots+\sqrt{d_{n}} \leq \frac{n}{2}\left(\frac{1}{\sqrt{n-d_{1}}}+\ldots+\frac{1}{\sqrt{n-d_{n}}}\right) .
$$

Now, from AM-QM for the positive numbers $\frac{1}{\sqrt{n-d_{1}}}, \ldots, \frac{1}{\sqrt{n-d_{n}}}$, and from (9) we get

$$
E(G) \leq \frac{n}{2}\left(\frac{1}{\sqrt{n-d_{1}}}+\ldots+\frac{1}{\sqrt{n-d_{n}}}\right) \leq \frac{n}{2} \sqrt{n\left(\frac{1}{n-d_{1}}+\ldots+\frac{1}{n-d_{n}}\right)} \leq \frac{n}{2} \sqrt{n \cdot \omega(G)} .
$$


Remark 2.5 In [15], Koolen and Moulton showed that $E(G) \leq \frac{n}{2}(1+\sqrt{n})$. For $\omega(G) \geq 2$ and $n>5$, we easily prove that $\frac{n}{2}(1+\sqrt{n})<\frac{n}{2} \sqrt{n \cdot \omega(G)}$. Thus, the bound in Theorem 2.4 is more useful in estimation of the clique number of a given graph through its order and its energy. Based on Theorem 2.4, for a graph $G$ on $n$ vertices and energy $E(G)$ it holds

$$
\omega(G) \geq \frac{4 E^{2}(G)}{n^{3}}
$$

The first 'trivial' upper bound for the energy of graph was obtained by McClelland [18], $E(G) \leq \sqrt{2 m n}$. We can come to this bound by using the upper bounds for the vertex energy and by using the inequality between quadratic and arithmetic mean. We have

$$
E(G)=E\left(v_{1}\right)+\ldots+E\left(v_{n}\right) \leq \sqrt{d_{1}}+\ldots+\sqrt{d_{n}} \leq \sqrt{n\left(d_{1}+\ldots+d_{n}\right)}=\sqrt{2 m n} .
$$

Now we extend this approach by fixing the maximum and the minimum degree of the graph. In [1], Alawiah et al. gave an upper bound on the energy of graphs, in terms of the size, maximum and minimum degree of the graph. In the following result we give a shorter proof of this theorem based on the upper bounds for the vertex energy.

Theorem 2.6 [1] Let $G$ be a non-empty graph with $n$ vertices, $m$ edges and maximum degree $\Delta$. Then

$$
E(G) \leq \sqrt{\Delta}+\sqrt{(n-1)(2 m-\Delta)}
$$

equality holds if and only if $G \equiv \frac{n}{2} K_{2}(n=2 m)$.

Proof. Without loss of generality we suppose that $\Delta=d_{1} \geq \ldots \geq d_{n}$ is the degree sequence of $G$. We have

$$
E(G)=E\left(v_{1}\right)+\ldots+E\left(v_{n}\right) \leq \sqrt{d_{1}}+\ldots+\sqrt{d_{n}}=\sqrt{\Delta}+\left(\sqrt{d_{2}}+\ldots+\sqrt{d_{n}}\right) .
$$

From the inequality between quadratic and arithmetic means for the numbers $\sqrt{d_{2}}, \ldots$, $\sqrt{d_{n}}$ we get

$$
\frac{\sqrt{d_{2}}+\ldots+\sqrt{d_{n}}}{n-1} \leq \sqrt{\frac{d_{2}+\ldots+d_{n}}{n-1}}=\sqrt{\frac{2 m-\Delta}{n-1}} .
$$

Now from (10) and (11) we get

$$
E(G) \leq \sqrt{\Delta}+\left(\sqrt{d_{2}}+\ldots+\sqrt{d_{n}}\right) \leq \sqrt{\Delta}+\sqrt{(n-1)(2 m-\Delta)} .
$$

Additionally, in [1] is given an upper bound for $E(G)$ for bipartite graphs, in terms of order, size, maximum and minimum degree. 
Theorem 2.7 [1] Let $G$ be a non-empty bipartite graph with $n \geq 2$ vertices, $m$ edges and maximum vertex $\Delta$. Then

$$
E(G) \leq 2 \sqrt{\Delta}+\sqrt{(n-2)(2 m-2 \Delta)}
$$

equality holds if and only if one of the following statements holds

(1) $G \cong \frac{n}{2} K_{2},(n=2 m)$;

(2) $K_{1, r-1} \cup\left(n-1-r_{n-1}\right) K_{1}$.

In the next theorem we give a similar bound as in Theorem 2.7. Note that our bound concerns all graphs, not only bipartite.

Theorem 2.8 For a graph $G$ with $n$ vertices, $m$ edges, maximum degree $\Delta$ and minimum degree $\delta$ it holds

$$
E(G) \leq \sqrt{\Delta}+\sqrt{\delta}+\sqrt{(n-2)(2 m-\Delta-\delta)}
$$

Proof. We proceed similarly as in Theorem 2.6. Let $\Delta=d_{1} \geq \ldots \geq d_{n}=\delta$ be the degree sequence of $G$. We get

$$
\begin{aligned}
E(G) & \leq \sqrt{d_{1}}+\ldots+\sqrt{d_{n}}=\sqrt{\Delta}+\sqrt{\delta}+\left(\sqrt{d_{2}}+\ldots+\sqrt{d_{n-1}}\right) \\
& \leq \sqrt{\Delta}+\sqrt{\delta}+\sqrt{(n-2)\left(d_{2}+\ldots+d_{n-1}\right)} \\
& =\sqrt{\Delta}+\sqrt{\delta}+\sqrt{(n-2)(2 m-\Delta-\delta)}
\end{aligned}
$$

Remark 2.9 Let us consider the function $f(x)=\sqrt{\Delta}+\sqrt{x}+\sqrt{(n-2)(2 m-\Delta-x)}$ defined on the interval $[\delta, \Delta]$. We calculate $f^{\prime}(x)=\frac{1}{2 \sqrt{x}}-\frac{n-2}{2 \sqrt{(n-2)(2 m-\Delta-x)}}$. Thus $f^{\prime}(x) \leq$ $0 \Leftrightarrow x \in\left[\frac{2 m-\Delta}{n-1}, \Delta\right]$. If $\delta \geq \frac{2 m-\Delta}{n-1}$, then $f$ is decreasing on the interval $[\delta, \Delta]$. In this case $f(\delta) \geq f(\Delta)$, that is, the bound in (12) is better than the bound in (13).

\subsection{New lower bounds}

Theorem 2.10 Let $G$ be a connected graph with $n$ vertices, $m$ edges and maximum degree

$\Delta$. Then

$$
E(G) \geq \frac{2 m}{\Delta}
$$


Proof. Let $e=\left(v_{i}, v_{j}\right) \in E(G)$. Since $E\left(v_{i}\right)+E\left(v_{j}\right) \geq 2$ we have

$$
\sum_{e \in E(G)}\left(E\left(v_{i}\right)+E\left(v_{j}\right)\right) \geq 2 m \Leftrightarrow d_{1} E\left(v_{1}\right)+\ldots+d_{n} E\left(v_{n}\right) \geq 2 m .
$$

From (15) we get

$$
E(G)=E\left(v_{1}\right)+\ldots+E\left(v_{n}\right)=\frac{\Delta \cdot\left(E\left(v_{1}\right)+\ldots+E\left(v_{n}\right)\right)}{\Delta} \geq \frac{d_{1} E\left(v_{1}\right)+\ldots+d_{n} E\left(v_{n}\right)}{\Delta} \geq \frac{2 m}{\Delta} .
$$

Remark 2.11 If $m \geq \Delta^{2}$, then $E(G) \geq \frac{2 m}{\Delta} \geq \frac{2 m}{\sqrt{m}}=2 \sqrt{m}$. In this case the bound (14) is better than the bound (5).

Remark 2.12 The bound in (14) straightforwardly follows from $E\left(v_{i}\right) \geq \frac{d_{i}}{\Delta}$, a result obtained in [3]. However, we decided to provide a different proof in order to present new relation between the vertex-degrees and vertex-energies.

Theorem 2.13 Let $G$ be a graph such that each vertex is contained in at least one triangle. If $n$ is the order of $G, \Delta$ is the maximum degree and $t$ is the number of triangles in the graph, then

$$
E(G) \geq \frac{36 t^{2}}{n \Delta^{2}(\Delta-1)^{2}}
$$

Proof. Let $V(G)=\left\{v_{1}, \ldots, v_{n}\right\}$ be the vertex set, $d_{1}, \ldots, d_{n}$ be the degree sequence of $G$ and let for each $i=1, \ldots, n$ the vertex $v_{i}$ be contained in $t_{i}$ triangles. Let $T(G)$ be the set of all triangles in $G$ and let $\left(v_{i}, v_{j}, v_{k}\right)$ be a triangle of $G$. Since $\left(v_{i}, v_{j}\right),\left(v_{i}, v_{k}\right)$ and $\left(v_{j}, v_{k}\right)$ are edges we get $E\left(v_{i}\right) \cdot E\left(v_{j}\right) \geq 1, E\left(v_{i}\right) \cdot E\left(v_{k}\right) \geq 1$ and $E\left(v_{j}\right) \cdot E\left(v_{k}\right) \geq 1$, which implies $E\left(v_{i}\right) E\left(v_{j}\right) E\left(v_{k}\right) \geq 1$. Thus and from Cauchy-Schwarz inequality we have

$$
\begin{gathered}
E(G)=E\left(v_{1}\right)+\ldots+E\left(v_{n}\right)=\sum_{\left(v_{i}, v_{j}, v_{k}\right) \in T(G)}\left(\frac{E\left(v_{i}\right)}{t_{i}}+\frac{E\left(v_{j}\right)}{t_{j}}+\frac{E\left(v_{k}\right)}{t_{k}}\right) \geq \\
\geq \sum_{\left(v_{i}, v_{j}, v_{k}\right) \in T(G)} \frac{3 \sqrt[3]{E\left(v_{i}\right) E\left(v_{j}\right) E\left(v_{k}\right)}}{\sqrt[3]{t_{i} t_{j} t_{k}}} \geq \sum_{\left(v_{i}, v_{j}, v_{k}\right) \in T(G)} \frac{9}{t_{i}+t_{j}+t_{k}} \geq \frac{9 t^{2}}{t_{1}^{2}+\ldots+t_{n}^{2}} .
\end{gathered}
$$

Since $t_{i} \leq\left(\begin{array}{c}d_{i} \\ 2\end{array}\right) \leq \frac{\Delta^{2}-\Delta}{2}$ we get $E(G) \geq \frac{36 t^{2}}{n \Delta^{2}(\Delta-1)^{2}}$.

We finish this section with a lower bound for the energy of hamiltonian graphs.

Theorem 2.14 If $G$ is a hamiltonian graph on $n$ vertices, then $E(G) \geq n$. 
Proof. Let $V(G)=\left\{v_{1}, \ldots, v_{n}\right\}$ be the vertex set of $G$. Since $G$ is a hamiltonian graph, there exists a permutation of $V(G)$, say $\left\{v_{1}^{\prime}, \ldots, v_{n}^{\prime}\right\}$, such that $v_{1}^{\prime}-v_{2}^{\prime}-\ldots-v_{n}^{\prime}-v_{1}^{\prime}$ is an $n$ - cycle. Hence, for each $i=1, \ldots, n$ holds

$$
E\left(v_{i}^{\prime}\right)+E\left(v_{i+1}^{\prime}\right) \geq 2 .
$$

Using the inequalities in (16), for the energy of $G$ we get

$$
E(G)=E\left(v_{1}^{\prime}\right)+\ldots+E\left(v_{n}^{\prime}\right)=\frac{1}{2}\left(\left(E\left(v_{1}\right)^{\prime}+E\left(v_{2}^{\prime}\right)\right)+\ldots+\left(E\left(v_{n}^{\prime}\right)+E\left(v_{1}^{\prime}\right)\right)\right) \geq n .
$$

\section{Energy of complement of a graph}

The complement of a graph $G$ is the graph $\bar{G}$ with vertex set $V(\bar{G})=V(G)$ and two vertices are adjacent in $\bar{G}$ if and only if they are not adjacent in $G$. More about the energy of the complementary graphs can be found in $[19,22]$. The following two wellknown results, obtained by Zhoe and Gutman, concern the bounds of $E(G)+E(\bar{G})$.

Theorem 3.1 [22] Let $G$ be a graph with $n$ vertices. Then

$$
E(G)+E(\bar{G}) \geq 2(n-1)
$$

with equality if and only if $G$ is the complete graph $K_{n}$ or its complement, the empty graph (the n-vertex graph without edges).

Theorem 3.2 [22] Let $G$ be a graph with $n$ vertices. Then

$$
E(G)+E(\bar{G})<\sqrt{2} n+(n-1) \sqrt{n-1} .
$$

As in the previous section, the results in this section rely on the bounds of the vertex energy. In the next two results we give new bounds on $E(G)+E(\bar{G})$.

Theorem 3.3 Let $G$ be a graph on $n$ vertices. Then

$$
E(G)+E(\bar{G}) \leq n \sqrt{2(n-1)}
$$

Proof. Let $d_{1}, \ldots, d_{n}$ be the degree sequence for the graph $G$. Clearly, $n-d_{1}-1, \ldots, n-$ $d_{n}-1$ is the degree sequence for the graph $\bar{G}$. We have

$$
E(G)+E(\bar{G}) \leq\left(\sqrt{d_{1}}+\ldots+\sqrt{d_{n}}\right)+\left(\sqrt{n-d_{1}-1}+\ldots+\sqrt{n-d_{n}-1}\right)
$$


Now, from the inequality between arithmetic and quadratic mean for the numbers $\sqrt{d_{i}}$ and $\sqrt{n-1-d_{i}}$ we get

$$
\sqrt{d_{i}}+\sqrt{n-1-d_{i}} \leq \sqrt{2\left(d_{i}+n-1-d_{i}\right)}=\sqrt{2(n-1)} .
$$

The relations in (17) and (18) yield

$$
E(G)+E(\bar{G}) \leq n \sqrt{2(n-1)}
$$

Remark 3.4 Doing elementary transformations we obtain that for $1 \leq n \leq 7$ it holds $n \sqrt{2(n-1)}<\sqrt{2} n+(n-1) \sqrt{n-1}$, that is, for $1 \leq n \leq 7$ the bound in Theorem 3.3 is better than the bound in Theorem 3.2.

In the next result we use the first Zagreb index of $G$, which is defined as $M_{1}(G)=$ $d_{1}^{2}+\ldots+d_{n}^{2}$.

Theorem 3.5 Let $G$ be a graph with $n$ vertices and $m$ edges. If $M_{1}(G)$ is the first Zagreb index of $G$, then

$$
E(G)+E(\bar{G}) \geq \frac{n^{2}(n-1)^{2}}{2 M_{1}(G)-4 m(n-1)+n(n-1)^{2}} .
$$

Proof. In [4] was proven that $E(G) \geq 2 R(G)$ and $E(\bar{G}) \geq 2 R(\bar{G})$. Thus

$$
\begin{aligned}
E(G)+E(\bar{G}) \geq 2(R(G)+R(\bar{G})) \\
=2\left(\sum_{e=\left(v_{i}, v_{j}\right) \in E(G)} \frac{1}{\sqrt{d_{i} d_{j}}}+\sum_{e=\left(v_{k}, v_{l}\right) \in E(\bar{G})} \frac{1}{\sqrt{\left(n-1-d_{k}\right)\left(n-1-d_{l}\right)}}\right) .
\end{aligned}
$$

From AM-GM for $d_{i}$ and $d_{j}$, and for $n-1-d_{k}$ and $n-1-d_{l}$, respectively, we obtain

$$
E(G)+E(\bar{G}) \geq 4\left(\sum_{e=\left(v_{i}, v_{j}\right) \in E(G)} \frac{1}{d_{i}+d_{j}}+\sum_{e=\left(v_{k}, v_{l}\right) \in E(\bar{G})} \frac{1}{\left(n-1-d_{k}\right)+\left(n-1-d_{l}\right)}\right) .
$$

Now, from Cauchy-Schwarz inequality we get

$$
\begin{aligned}
E(G)+E(\bar{G}) & \geq 4 \cdot \frac{(1+1+\ldots+1)^{2}}{d_{1}^{2}+\ldots+d_{n}^{2}+\left(n-1-d_{1}\right)^{2}+\ldots+\left(n-1-d_{n}\right)^{2}} \\
& =\frac{4\left(\frac{n(n-1)}{2}\right)^{2}}{2\left(d_{1}^{2}+\ldots+d_{n}^{2}\right)-4 m(n-1)+n(n-1)^{2}} \\
& =\frac{n^{2}(n-1)^{2}}{2 M_{1}(G)-4 m(n-1)+(n-1)^{2} n} .
\end{aligned}
$$


Theorem 3.6 Let $G$ be a graph with $n$ vertices, $m$ edges and minimum degree $\delta$. Then

$$
\sqrt{n\left(n^{2}-2 m-n\right)} \geq E(\bar{G}) \geq \frac{n^{2}-n-2 m}{n-1-\delta} .
$$

Proof. Let $d_{1} \geq d_{2} \geq \ldots \geq d_{n}=\delta$ be the degree sequence of $G$. We observe that $\bar{G}$ has $\frac{n(n-1)}{2}-m=\frac{n^{2}-n-2 m}{2}$ edges. Let $e=\left(v_{i}, v_{j}\right) \in E(\bar{G})$. Then $E\left(v_{i}\right)+E\left(v_{j}\right) \geq 2$. Applying the same idea as in Theorem 2.10 we have

$\sum_{e \in E(\bar{G})}\left(E\left(v_{i}\right)+E\left(v_{j}\right)\right) \geq n^{2}-n-2 m \Leftrightarrow\left(n-1-d_{1}\right) E\left(v_{1}\right)+\ldots+\left(n-1-d_{n}\right) E\left(v_{n}\right) \geq n^{2}-n-2 m$.

Since $n-1-\delta$ is the maximum degree of $\bar{G}$, from (19) we have

$$
\begin{aligned}
E(G) & =\frac{(n-1-\delta)\left(E\left(v_{1}\right)+\ldots+E\left(v_{n}\right)\right)}{n-1-\delta} \\
& \geq \frac{\left(n-1-d_{1}\right) E\left(v_{1}\right)+\ldots+\left(n-1-d_{n}\right) E\left(v_{n}\right)}{n-1-\delta} \geq \frac{n^{2}-n-2 m}{n-1-\delta} .
\end{aligned}
$$

The upper bound for $E(\bar{G})$ is derived as follows

$$
\begin{aligned}
E(\bar{G}) & \leq \sqrt{n-1-d_{1}}+\ldots+\sqrt{n-1-d_{n}} \leq \sqrt{n\left(\left(n-1-d_{1}\right)+\ldots+\left(n-1-d_{n}\right)\right)} \\
& =\sqrt{n\left(n^{2}-n-2 m\right)} .
\end{aligned}
$$

\section{References}

[1] N. Alawiah, N. J. Rad, A. Jahanbani, H. Kamarulhaili, New upper bounds on the energy of a graph, MATCH Commun. Math. Comput. Chem. 79 (2018) 287-301.

[2] O. Arizmendi, O. Juarez-Romero, On bounds for the energy of graphs and digraphs, in: F. Galaz-García, J. C. P. Millán, P. Solórzano (Eds.), Contributions of Mexican Mathematicians Abroad in Pure and Applied Mathematics, Am. Math. Soc., Providence, 2018, pp. 1-19.

[3] O. Arizmendi, J. F. Hidalgo, O. Juarez-Romero, Energy of a vertex, Lin. Algebra Appl. 557 (2018) 464-495.

[4] G. Arizmendi, O. Arizmendi, Energy of a graph and Randić index, Lin. Algebra and Appl. 609 (2021) 332-338.

[5] D. Babić, I. Gutman, More lower bounds for the total $\pi$-electron energy of alternant hydrocarbons, MATCH Commun. Math. Comput. Chem. 32 (1995) 7-17.

[6] G. Caporossi, D. Cvetković, I. Gutman, P. Hansen, Variable neighborhood search for extremal graphs. 2. Finding graphs with extremal energy, J. Chem. Inf. Comput. Sci. 39 (1999) 984-996. 
[7] Y. Caro, New results on the independence number, Technical report, Tel Aviv Univ., 1979.

[8] I. Gutman, The energy of a graph, Ber. Math. Stat. Sekt. Forschungsz. Graz, 103 (1978) 1-22.

[9] I. Gutman, The energy of a graph: old and new results, in: A. Betten, A. Kohnert, R. Laue, A. Wassermann (Eds.), Algebraic Combinatorics and Applications, Springer, Berlin, 2001, pp. 196-211.

[10] I. Gutman, O. E. Polansky, Mathematical Concepts in Organic Chemistry, Springer, Berlin, 1986.

[11] I. Gutman, On graphs whose energy exceeds the number of vertices, Lin. Algebra Appl. 429 (2008) 2670-2677.

[12] I. Gutman, X. Li, J. Zhang, Graph energy, in: M. Dehmer, F. Emmert-Streib (Eds.), Analysis of Complex Networks. From Biology to Linguistics, Wiley-VCH, Weinheim, 2009, pp. 145-174.

[13] I. Gutman, A. V. Teodorović, L. Nedeljković, Topological properties of benzenoid systems. Bounds and approximate formula for total $\pi$-electron energy, Theor. Chim. Acta. 65 (1984) 23-31.

[14] A. Jahanbani, Some new lower bounds for energy of graphs, Appl. Math. Comput. 296 (2017) 233-238.

[15] J. H. Koolen, V. Moulton, Maximal energy graphs, Adv. Appl. Math. 26 (2001) 47-52.

[16] J. H. Koolen, V. Moulton, I. Gutman, Improving the McClelland inequality for total $\pi$-electron energy, Chem. Phys. Lett. 320 (2000) 213-216.

[17] X. Li, Y. Shi, I. Gutman, Graph Energy, Springer, New York, 2010.

[18] B. J. McClelland, Properties of the latent roots of a matrix: The estimation of $\pi$ electron energies, J. Chem. Phys. 54 (1971) 640-643.

[19] H. S. Ramane, B. Parvathalu, D. D. Patil, K. Ashoka, Graphs equienergetic with their complements, MATCH Commun. Math. Comput. Chem. 82 (2019) 471-480.

[20] V. K. Wei, A lower bound on the stability number of a simple graph, Technical Report 81-11217-9, Bell Labs., 1981.

[21] Z. Yan, C. Liu, Y. Pan, J. Li, Energy, Randić index and maximum degree of graphs, MATCH Commun. Math. Comput. Chem. 86 (2021) 539-542. 
[22] B. Zhou I. Gutman, Nordhaus-Gaddum-type relations for the energy and Laplacian energy of graphs, Bull. Acad. Serbe Sci. Arts. Class. Sci. Math. Nat. 32 (2007) 1-11. 